\title{
A CONTRIBUIÇÃO DE C. KUHLTHAU PARA A CIÊNCIA DA INFORMAÇÃO NO BRASIL
}

\author{
Carlos Alberto Ávila Araújo \\ Rogério Manoel de Oliveira Braga \\ Wellington de Oliveira Vieira
}

\section{Resumo:}

O artigo apresenta os resultados de uma pesquisa que buscou ver o impacto de Kuhlthau na produção científica brasileira em Ciência da Informação. Buscou-se referências à autora em todos os artigos publicados em sete periódicos nacionais, entre os anos de 2003 e 2007. Kuhlthau é citada em 17 dos 872 artigos analisados. Das 19577 referências bibliográficas destes artigos, 24 são de trabalhos de Kuhlthau. Essas referências foram analisadas buscando-se identificar a freqüência de cada uma das obras da autora citadas, ano, idioma, natureza e casos de co-autoria. Os artigos citantes foram analisados em termos de temática e procedência institucional dos autores. Por fim, analisou-se a importância das citações à autora para as discussões empreendidas nos artigos citantes. Entre os resultados encontrados destacam-se: a obra mais citada, Inside the search process: information seeking from the user's perspective, teve 8 referências; a maior contribuição da autora se dá no âmbito dos estudos de usuários da informação; a instituição brasileira que mais a cita é a UFMG; e o tipo de citação mais freqüente é a citação conceitual.

\section{Palavras-chave:}

Carol Kuhlthau; Pesquisa brasileira em Ciência da Informação; Estudo bibliométrico

\section{KUHLTHAU CONTRIBUTION FOR INFORMATION SCIENCE IN BRAZIL}

\begin{abstract}
:
This article presents the results of a research about the impact of Kuhlthau in the Brazilian production of Information Science. It was taken citations of the author in all articles published for seven Brazilian journals, from 2003 to 2007. Kuhlthau is cited in 17 of the 872 articles analyzed. From the 19,577 bibliographic references of these articles, 24 are Kuhlthau's works. These references were analyzed to identify the frequency of each Kuhlthau's work cited, year, language, kind and co-authorship cases. The citing articles were analyzed in terms of thematic and institutional origin of the authors. Finally, it was examined the importance of the citation of the author for the discussions in citing articles. Some results are: the most cited work, Inside the search process: information seeking from the user's perspective, with 8 references; the author's greatest contribution is in the information's user studies; the Brazilian institution that most cite Kuhlthau works is UFMG, and, the most important kind of citation is the conceptual citation.
\end{abstract}

\section{Keywords:}

Carol Kuhlthau; Brazilian research in Information Science; Bibliometrics 


\section{Introdução}

A pesquisa científica em Ciência da Informação no Brasil vem crescendo consideravelmente nas últimas décadas. Vários periódicos científicos têm sido criados, ao longo dos anos, para favorecer a divulgação dos trabalhos na área e a comunicação entre os pesquisadores brasileiros. A importância da pesquisa científica em Ciência da Informação está fato de que por meio dela é possível desenvolver novos conceitos, teorias e métodos e, conseqüentemente, o aperfeiçoamento e crescimento dessa ciência. Qualquer área do conhecimento, até chegar à maturidade de suas teorias e se consolidar como ciência, passa por uma série de alavancagens teórico-metodológicas.

Uma das formas de se contribuir para o incremento da pesquisa em Ciência da Informação no Brasil é descobrir quais são as maiores influências, em termos de pesquisadores, e como vêm se dando essas contribuições para a área no país. Por meio de pesquisas realizadas no Brasil foi identificada uma importante pesquisadora norteamericana, chamada Carol Collier Kuhlthau, que vem trazendo grandes contribuições para a CI no país. Como se verá mais adiante, a autora é uma das precursoras na área de busca da informação e seu modelo sobre o processo de busca de informações é referenciado na maior parte dos trabalhos que incluem estudo de usuários.

Este artigo apresenta o resultado de uma pesquisa que buscou avaliar a maneira como vem se dando a contribuição dessa pesquisadora na produção brasileira em Ciência da Informação. Em um levantamento realizado com pesquisadores brasileiros da área de Ciência da Informação (ARAÚJO et al, 2007a), diante de uma pergunta sobre quem são os principais autores do campo, Kuhlthau figurou entre os trinta autores mais citados pelos respondentes, numa lista de 137 autores mencionados. Em outra pesquisa, dessa vez realizada com professores da Escola de Ciência da Informação da UFMG (ARAÚJO et al, 2007b), diante da mesma pergunta, Kuhlthau foi a décima-primeira autora mais citada. Neste caso, os respondentes citaram 107 autores.

Os resultados dos dois levantamentos apontaram para uma relativa importância dessa pesquisadora, no que diz respeito ao reconhecimento dado a ela pelos pesquisadores brasileiros. Contudo, esse resultado acabou por provocar algumas inquietações: como estaria sendo o efetivo impacto dessa autora na produção científica brasileira? Que 
trabalhos dela são citados? Que instituições brasileiras mais a citam? Que idéias dela são utilizadas e com que importância para as pesquisas realizadas?

Para responder a essas questões, e como forma de dar continuidade às duas pesquisas mencionadas acima, foi realizada uma pesquisa com todos os artigos publicados entre os anos de 2003 e 2007 em sete periódicos brasileiros: Ciência da Informação (Instituto Brasileiro de Informação em Ciência e Tecnologia - IBICT), Perspectivas em Ciência da Informação (Universidade Federal de Minas Gerais - UFMG), Informação \& Sociedade: Estudos (Universidade Federal da Paraíba - UFPb), Datagramazero (Instituto de Adaptação e Inserção na Sociedade da Informação - IASI/RJ), Transinformação (Pontifícia Universidade Católica de Campinas - Puccamp), Encontros Bibli (Universidade Federal de Santa Catarina - UFSC) e Em Questão (Universidade Federal do Rio Grande do Sul - UFRGS). Foram escolhidos estes periódicos pois eram, no início da pesquisa, aqueles classificados como “periódico nacional” no critério Qualis da Capes (Coordenação de Aperfeiçoamento de Pessoal de Nível Superior).

O trabalho de análise se deu da seguinte forma: inicialmente, foram consultados todos os artigos publicados nos periódicos e selecionados aqueles que tinham referências de Kuhlthau, para a composição de um "banco de artigos citantes”. A partir da análise desse banco, foram identificadas as obras de Kuhlthau que são citadas e sua identificação por ano de publicação, natureza do trabalho, língua e casos de co-autoria. A seguir, foram analisados os artigos citantes, em termos de temática e de procedência institucional dos autores. Por fim, foram analisadas, artigo por artigo, as citações feitas à autora, avaliando a sua contribuição para a discussão empreendida nos artigos correspondentes. A partir do conjunto dos dados coletados e analisados, buscou-se caracterizar, então, a contribuição desta autora para a pesquisa brasileira em Ciência da Informação.

\section{Sobre a autora}

Carol Collier Kuhlthau é professora titular da School of Communication, Information and Library Studies, da Rutgers University (New Jersey, EUA), desde 1985. Concluiu seu bacharelado na faculdade de Kean, o mestrado e doutorado na Universidade de Rutgers. Já exerceu importantes atividades profissionais em várias bibliotecas norte-americanas e

(c) Revista Digital de Biblioteconomia e Ciência da Informação,Campinas, v.7, n. 2, p. 185-198, jan.jjun. 2010- ISSN: 1678-765X. 
também cargos em diversas instituições como diretora, consultora, pesquisadora, professora, coordenadora e conferencista, dentre outras.

Seu interesse de pesquisa se refere à perspectiva do usuário no processo de busca da informação. Suas pesquisas incluem também educação de usuário, aspectos cognitivos e afetivos envolvidos no processo de busca da informação e serviços de biblioteca centrados no usuário. Kuhlthau já escreveu 12 livros, 19 capítulos de livros, 57 artigos científicos, quatro críticas de livros e nove outros tipos de publicações. Foi conferencista em diversos eventos internacionais e participa de forma ativa de associações profissionais.

A autora é uma das pioneiras nesta área de busca da informação e o modelo desenvolvido por ela sobre o processo de busca da informação é referenciado na maior parte dos trabalhos que envolvem estudo de usuários. Esse modelo se destacou por concentrar os aspectos afetivos, cognitivos e físicos presentes nos indivíduos no momento de procura e uso da informação. Os pensamentos, as ações e os sentimentos que, geralmente, são vivenciados pelos usuários em cada estágio do processo de busca da informação são descritos neste modelo.

Entre suas experiências profissionais, destacam-se a atuação como bibliotecária em North Brunswick, entre 1975 e 1985 e sua atuação como professora visitante na Faculty of Education da University of Hong Kong (2006), no Department of Information Management, Aberdeen Business School, Aberdeen, Escócia, (2007) e como pesquisadora senior no Department of Information Sciences da University Strossmayer, Osijek, Croácia, (2006). Dentre as atividades institucionais, destacam-se a cátedra no departamento de Library and Information Science, entre os anos de 1999 e 2002, e a diretoria do Center for International Scholarship in School Libraries (CISSL) de 2003 a 2006 (RUTGERS..., 2008).

Kuhlthau já recebeu vários prêmios de diversas instituições importantes ligadas à área e já foi convidada para participar de seminários, congressos e palestras em diversas universidades no mundo inteiro, tais como Tampere (Finlândia), Gotemburgo (Suécia), Copenaguen (Dinamarca), Taipei (Tailândia), Kyoto (Japão) entre muitas outras. A autora já esteve no Brasil. Atualmente, ela é homenageada por meio do "Prêmio Carol 
Kuhlthau”, instituído pelo Grupo de Estudos em Biblioteca Escolar da UFMG em parceria com a Autêntica Editora e destinado a profissionais brasileiros que atuam em bibliotecas do ensino básico.

\section{Referências feitas à autora}

A primeira parte da pesquisa consistiu no rastreamento de artigos que citassem a autora. Do total de 872 artigos publicados nos sete periódicos brasileiros estudados, no período determinado, 17 tinham referência a alguma obra de Kuhlthau - o que equivale a 1,94\% do total da produção científica do período. Nestes 872 artigos foram encontradas 19577 referências bibliográficas. Destas, 24 referem-se a obras de Kuhlthau. A Tabela 1, a seguir, apresenta a contagem geral de todas as referências bibliográficas presentes nos artigos analisados, separadas por ano e por periódico. Apresenta, ainda, a contagem das referências a Kuhlthau.

TABELA 1 - Somatório das referências, por ano e periódico

\begin{tabular}{c|c|c|c|c|c|c|c|c|c|c|c|c}
\hline \multirow{2}{*}{ Periódico } & \multicolumn{2}{|c|}{2003} & \multicolumn{2}{c|}{2004} & \multicolumn{2}{c|}{2005} & \multicolumn{2}{c|}{2006} & \multicolumn{2}{c|}{2007} & \multicolumn{2}{c}{ TOTAL } \\
\cline { 2 - 12 } & A & T & A & T & A & T & A & T & A & T & A & T \\
\hline CI & 3 & 741 & 0 & 1033 & 0 & 682 & 2 & 1240 & 4 & 851 & $\mathbf{9}$ & 4547 \\
PCI & 1 & 552 & 0 & 323 & 2 & 321 & 0 & 531 & 0 & 663 & $\mathbf{3}$ & 2390 \\
DAT & 0 & 593 & 0 & 652 & 0 & 609 & 0 & 765 & 3 & 773 & $\mathbf{3}$ & 3392 \\
TRA & 1 & 640 & 0 & 366 & 0 & 417 & 0 & 419 & 0 & 474 & $\mathbf{1}$ & 2316 \\
I\&S & 0 & 350 & 0 & 361 & 0 & 326 & 1 & 760 & 0 & 592 & $\mathbf{1}$ & 2389 \\
EBI & 0 & 109 & 1 & 457 & 0 & 320 & 1 & 946 & 1 & 738 & $\mathbf{3}$ & 2570 \\
EMQ & 4 & 326 & 0 & 423 & 0 & 402 & 0 & 493 & 0 & 329 & $\mathbf{4}$ & 1973 \\
\hline TOTAL & $\mathbf{9}$ & $\mathbf{3 3 1 1}$ & $\mathbf{1}$ & $\mathbf{3 6 1 5}$ & $\mathbf{2}$ & $\mathbf{3 0 7 7}$ & $\mathbf{4}$ & $\mathbf{5 1 5 4}$ & $\mathbf{8}$ & $\mathbf{4 4 2 0}$ & $\mathbf{2 4}$ & $\mathbf{1 9 5 7 7}$ \\
\hline
\end{tabular}

NOTA: Na tabela acima, os periódicos são identificados pelas siglas CI (Ciência da Informação), PCI (Informação \& Sociedade: Estudos), EBI (Encontros Bibli) e EMQ (Em Questão). Nas colunas relativas aos anos, a coluna identificada com a letra "A" indica a quantidade de referências a Kuhlthau naquele periódico naquele ano, e a coluna " $T$ ” indica o total de referências bibliográficas de todos os artigos publicados naquele periódico naquele ano. 
A tabela acima mostra que não existe uma regularidade na distribuição das referências

bibliográficas. As maiores incidências de citações à autora se deram nos extremos, isto é, nos anos de 2003 (nove citações) e 2007 (oito citações), sendo os anos de 2004 e 2005 aqueles nos quais se verificou a menor incidência.

Já em relação aos periódicos, nota-se que, em termos absolutos, sua maior incidência se dá na revista Ciência da Informação, com nove ocorrências, seguida de Em Questão, com quatro. Em termos percentuais, porém, a maior incidência se dá em Em Questão (0,20\%), seguindo-se Ciência da Informação (0,19\%) e Perspectivas em Ciência da Informação $(0,12 \%)$.

\section{Características das obras citadas}

A análise seguinte recaiu sobre as obras de Kuhlthau citadas nos artigos. Um total de 10 obras da autora foram citadas, como apresentado a seguir, na Tabela 2: 
TABELA 2 - Obras de Kuhlthau citadas nos artigos

\section{Obras da autora citadas}

KUHLTHAU, Carol C. Inside the Search Process: information seeking from the user's perspective. Journal of The American Society for Information Science, New York, v.42, n.5, p.361-371, 1991.

KUHLTHAU, Carol C. Seeking meaning: a process approach to library and information services. Norwood, N.J.: Ablex, 1996. (incluindo todas as edições posteriores citadas)

KUHLTHAU, Carol C. A principle of uncertainty for information seeking. Journal of Documentation, v. 49, n. 4, p. 339-355, 1993.

KUHLTHAU, Carol C. Como usar a biblioteca na escola: um programa de atividades para o ensino fundamental. Trad. e Adapt. por Bernadete Campello dos Santos et al. Belo Horizonte: Autêntica, 2002.

KUHLTHAU, Carol C. O papel da biblioteca escolar no processo de aprendizagem. In: VIANNA, M. M. et al. Seminário Biblioteca Escolar: espaço de ação pedagógica. Belo Horizonte: Escola de Ciência da Informação da UFMG, 1999. p. 9-14.

KUHLTHAU, Carol C.; TAMA, S. L. Information search process of lowers: a call for "just for me" information service. Journal of Documentation, London, v. 57, n. 1, p. 25-43, Jan. 2001.

KUHLTHAU, Carol C. The process of learning from information. In: The Virtual School Library: gateway to the information superhighway. Englewood: Libraries, p. 95104, 1996.

KUHLTHAU, Carol C. The role of experience in the information search process of an early career information worker: perceptions of uncertanty, complexity, construction, and sources. Journal of the American Society for Information Science, Washington, DC, v. 50, n. 5, p. 399412, Apr. 1999.

KUHLTHAU, Carol C. Learning in digital libraries: an information search process approach. Library Trends, v. 45, n. 4, p. 708-724, 1997.

KUHLTHAU, Carol C. Student and the information search process: zones of intervention for librarians. Advances in Librarianship, New York, v.18, p.57-72, 1994.

\section{Freqüência}

8

5

2 
Percebe-se assim uma variedade de obras da autora utilizadas na pesquisa brasileira. A obra mais citada da autora, o artigo Inside the search process: information seeking from the user's perspective é referenciado oito vezes, perfazendo 33,33\% das referências feitas à autora. Somando-se a segunda obra mais referenciada, o livro Seeking meaning: $a$ process approach to library and information services, com cinco referências, tem-se um total de 13 referências, ou seja, 54,16 \% das referências. Um pouco menos da metade das referências feitas à autora (45,84\%) espalha-se por outras oito obras.

Os trabalhos da autora, referenciados nos trabalhos científicos brasileiros no período determinado, cobrem um vasto período de tempo, indo de 1991 a 2002. Percebe-se, contudo, que a maior parte das referências são de trabalhos do início da década de 1990: sua contribuição se concentra em obras publicadas entre os anos de 1991 e 1996. Somando-se as referências a obras publicadas neste período, tem-se o total de 17 referências, perfazendo 70,83\%. Em relação à natureza das obras, tem-se que 14 citações recebidas referem-se a artigos publicados em periódicos (58,33\%), sendo outras 10 referentes a livros e capítulos de livro.

Quanto ao idioma, percebe-se que 20 referências são de obras da autora em inglês (83,33\%), principalmente relacionadas aos seus trabalhos da década de 1990. As quatro citações recebidas por trabalhos em português são, justamente, mais recentes e, talvez por esse motivo, tenham tido menos citações. Por fim, entre as referências feitas a trabalhos da autora, apenas uma se refere a trabalhos realizados em co-autoria.

\section{Sobre os artigos citantes}

A análise seguinte recaiu sobre os 17 artigos que fizeram referência a obras de Kuhlthau. Essa análise se deu em dois momentos. Inicialmente, foi analisada a temática do artigo. Para isso, foram contabilizadas todas as palavras-chave presentes em cada um deles. Como resultado, foram encontradas 48 diferentes palavras-chave, conformando uma relativa dispersão.

A palavra mais freqüente foi “competência informacional”, com seis incidências, sendo que ainda se verificou uma ocorrência para "competência em informação" e outra para “competências informacionais” - totalizando oito. A seguir aparece "busca de 
informação” com cinco incidências, além de uma para "búsqueda de información”, perfazendo seis ocorrências.

“Comportamento informacional” é o terceiro termo mais freqüente, com três ocorrências, havendo também uma para "comportamento de busca da informação” e “comportamiento en La búsqueda de información”. Também com três ocorrências aparece "biblioteca escolar”. Apareceram duas vezes “uso de informação”, "bibliotecário”, “information literacy”, “necessidades de informação” e “ciência da informação”.

Este quadro, somado à presença de outros termos com apenas uma freqüência sem trema, mostra bem o tipo de universo temático vinculado à autora: em primeiro lugar destacamse os comportamentos informacionais, busca, necessidade, uso; a seguir, as áreas de competência e aprendizagem, ligados normalmente à biblioteca e à biblioteca escolar. Mas também apareceram termos ligados à área médica, comunicação científica, biologia molecular, interação humano-computador, cognição, entre outras.

Contudo, apenas a indicação das palavras-chave, dada sua grande variação, não fornece um indicador preciso das temáticas efetivamente impactadas pelos trabalhos de Kuhlthau. Para se ter uma visão mais precisa trabalhou-se na perspectiva de identificação das principais teorias às quais os artigos analisados se vinculam. Para tanto, utilizou-se um quadro de referência (ARAÚJO, 2008) que prevê as seguintes categorias:

a) Estudos inspirados na teoria matemática, centrados no transporte físico da informação;

b) Estudos de natureza sistêmica;

c) Estudos críticos da informação;

d) Estudos sobre representação, classificação, catalogação;

e) Estudos sobre a comunicação científica, fluxos, redes;

f) Estudos de usuários da informação;

g) Estudos bibliométricos.

A aplicação deste quadro de referência sobre os 17 artigos mostrou que a maior parte destes encontra-se vinculado às temáticas dos estudos de usuários, com 10 incidências (58,82\%). Outras duas temáticas que tiveram destaque foram a comunicação científica,

(c) Revista Digital de Biblioteconomia e Ciência da Informação,Campinas, v.7, n. 2, p. 185-198, jan.jjun. 2010- ISSN: 1678-765X. 
com três referências $(17,64 \%)$ e a teoria sistêmica, com duas (11,76\%), sendo que ainda foi possível identificar um artigo vinculado à teoria matemática e outro à teoria crítica.

Analisou-se também a procedência institucional dos autores dos artigos. Para tanto, considerou-se a técnica bibliométrica de se contabilizar apenas uma freqüência para cada artigo, independente do número de autores (FIGUEIREDO, 1977; VANTI, 2002; ROUSSEAU, 1998). Assim, no caso de artigos com autores pertencentes a mais de uma instituição, a contagem foi parcelada, isto é, dividida entre as instituições envolvidas na produção do artigo. Por exemplo, no caso de um artigo produzido por dois autores, cada um de uma instituição, foi atribuído 0,5 ponto para cada instituição. E assim sucessivamente.

Para a definição da instituição, considerou-se em primeiro lugar o vínculo profissional, quando este se relacionava a instituição de ensino superior e/ou de pesquisa. Quando não foi o caso, considerou-se a vinculação de formação acadêmica, isto é, onde o autor realizou seu doutorado ou mestrado. Apenas quando não foi possível considerar estas duas condições, contabilizou-se a instituição de pertencimento profissional. Ressalte-se que houve um volume considerável de casos em que os autores não indicavam qualquer vínculo institucional.

Uma vez completada a contabilização, identificou-se que a instituição de onde provém a maior parte dos artigos que citam Kuhlthau é a UFMG, que atingiu o total de 6,5 artigos, seguida da UFRGS, com três, e USP, com dois. No total foram encontradas, além destas, outras seis instituições às quais pertencem os autores dos artigos citantes.

\section{Importância das citações feitas a Kuhlthau}

A próxima etapa da pesquisa consistiu na análise dos textos das citações de Kuhlthau presentes nos artigos, buscando avaliar a importância das idéias da autora para a discussão empreendida no artigo. Para a avaliação dessa importância, as citações foram classificadas a partir das seguintes categorias:

(c) Revista Digital de Biblioteconomia e Ciência da Informação,Campinas, v.7, n. 2, p. 185-198, jan./jun. 2010- ISSN: 1678-765X. 
- Citação conceitual: traz a definição de algum conceito trabalhado no artigo;

- Citação metodológica: apresenta os passos ou procedimentos para a execução de alguma atividade ou pesquisa;

- Citação exemplificativa: traz outros casos, realidades ou estudos para demonstrar algum ponto ou questão;

- Citação confirmativa: dá suporte a alguma idéia ou afirmação;

- Citação negativa/crítica: utilizada para contrapor alguma idéia ou afirmação;

- Citação de sustentação: usada para embasar ou dar suporte a alguma idéia, com dados ou outras idéias;

- Citação panorâmica/de revisão: objetiva dar um panorama de quem mais estudou determinada questão;

- Citação orgânica/de compreensão: necessária para a compreensão do que está sendo afirmado;

As categorias acima definidas foram aplicadas a cada uma das 73 citações à autora (contando-se as várias vezes em que, dentro de um mesmo artigo, trechos ou idéias de Kuhlthau foram citados, de forma direta ou indireta) presentes nos 17 artigos citantes. A maior incidência foi de citações conceituais, num total de 22. Nestas, observa-se que os autores brasileiros buscam, nos trabalhos da autora, as definições de "incerteza", “construtivismo”, “letramento”, entre outras. Em segundo lugar aparecem as citações de sustentação, com 11 incidências. São trabalhos que realizam pesquisas utilizando as categorias de Kuhlthau, isto é, usando o esquema teórico da autora como base para a realização de pesquisas ou discussões específicas.

Com dez incidências encontram-se as citações exemplificativas. Nestes casos, normalmente reporta-se a resultados de pesquisa e achados da autora para exemplificar a maneira como acontecem determinados processos ou fenômenos (na quase totalidade dos casos, associados aos processos de busca de informação). Com nove incidências estão as citações panorâmicas: Kuhlthau é normalmente citada nas revisões de literatura, apontada, junto com autores como Belkin, Dervin e Taylor, como representante da abordagem “alternativa” ou cognitiva de estudos de usuários da informação. Destacam-se ainda oito citações de natureza metodológica, isto é, que apresentam a maneira com que a autora conduz seus estudos empíricos e/ou tentam replicar sua metodologia em estudos realizados no Brasil. Também oito são as citações confirmativas, que desenvolvem 
alguma idéia (por exemplo, sobre os seis estágios do processo de busca de informação, ou sobre a colaboração entre o professor e o bibliotecário no ensino básico) e utilizam a autora apenas para comprovar essa idéia.

\section{Considerações finais}

Este trabalho teve como finalidade realizar uma análise crítica e bibliométrica da contribuição de Carol Collier Kuhlthau para a Ciência da Informação no Brasil, com base em varreduras feitas em sete periódicos brasileiros da área.

Por meio deste trabalho foi possível identificar seus trabalhos mais citados (o artigo Inside the Search Process: information seeking from the user's perspective e o livro Seeking meaning: a process approach to library and information services), as revistas brasileiras que mais a citam (Ciência da Informação e Em Questão), a instituição de onde provêm o maior número de autores que mais a citam (UFMG).

Em relação às temáticas dos trabalhos que a citam, nota-se uma predominância de temas como a competência informacional e os comportamentos informacionais (necessidades, busca e uso de informação), além de questões ligadas à biblioteca escolar. Sua contribuição se dá no âmbito de diferentes teorias mas, sobretudo, nos estudos de usuários.

No Brasil, o principal impacto da abordagem de busca por informação por crianças e adolescentes abordado por Kuhlthau está relacionado ao papel da biblioteca escolar no processo de aprendizagem. Idéias e resultados de pesquisa de Kuhlthau contribuíram para que os pesquisadores brasileiros trabalhassem na perspectiva do conceito de “competência informacional”, ou seja, a habilidade do usuário de construir sentido por si mesmo, em um ambiente rico em informação.

Podemos perceber também que, com o apoio dos trabalhos de Kuhlthau, os estudos sobre usuários no Brasil passaram de uma orientação voltada para os sistemas (onde a informação é objetiva e a principal questão é como obtê-la), para uma orientação centrada no usuário, em suas tarefas e contextos concretos. O modelo teórico abordado por Kuhlthau teve e tem grande importância no Brasil, pois trata a informação como algo subjetivo, que só se torna útil para o usuário quando este cria um sentido para ela. Dessa 
maneira, os estudos de usuários no contexto brasileiro vêm se voltando para uma pesquisa mais qualitativa e focada no usuário, com especial atenção para as etapas percorridas no processo de busca da informação, componente essencial do comportamento informacional. E esse movimento tem ocorrido com a contribuição fundamental de Carol Kuhlthau.

\section{Referências}

ARAÚJO, Carlos Alberto Ávila et al. A ciência da informação na visão dos professores e pesquisadores brasileiros. Informação \& Sociedade: Estudos, João Pessoa, v. 17, n.2, p. 110-127, maio/ago. 2007a.

ARAÚJO, Carlos Alberto Ávila et al. A ciência da informação na visão dos professores da ECI/UFMG. Perspectivas em Ciência da Informação, Belo Horizonte, v. 12, n.2, p. 3-22, maio/ago. 2007b.

ARAÚJO, Carlos Alberto Ávila. Teorias da ciência da informação. Belo Horizonte: Escola de Ciência da Informação da UFMG, 2008. Relatório de pesquisa.

FIGUEIREDO, Nice. Tópicos modernos em bibliometria. Brasília: Associação dos Bibliotecários do Distrito Federal, 1977.

ROUSSEAU, Ronald. Indicadores bibliométricos e econométricos para a avaliação de instituições científicas. Ciência da Informação, Brasília, v. 27, n. 2, p. 149-158, maio/ago. 1998.

RUTGERS University. Carol Collier Kuhlthau. New Jersey, 2008. Disponível em: http://www.scils.rutgers.edu/ kuhlthau/. Acesso em: 19 abr. 2009.

VANTI, Nadia Aurora. Da bibliometria à webometria: uma exploração conceitual dos mecanismos utilizados para medir o registro da informação e a difusão do conhecimento. Ciência da Informação, Brasília, v. 31, n. 2, p. 152-162, maio/ago. 2002. 


\section{Carlos Alberto Ávila Araújo}

Professor adjunto da Escola de Ciência da Informação da UFMG. Doutor em Ciência da Informação. casalavila@yahoo.com.br

\section{Rogério Manoel de Oliveira Braga}

Bacharel em Biblioteconomia pela Escola de Ciência da Informação da UFMG. Agente Administrativo da Ouvidoria Geral do Estado de Minas Gerais. rogermoby@gmail.com

\section{Wellington de Oliveira Vieira}

Bacharel em Biblioteconomia pela Escola de Ciência da Informação da UFMG. Auxiliar Administrativo Tecnometal Engenharia e Construções Mecânicas Ltda. wellbiblio@gmail.com

Recebido em: 30/04/2009

Aceito para publicação em: jul/2009 